\title{
La relación entre fumar y depresión en la adolescencia: Implicaciones clínicas y preventivas
}

\author{
BecoñA, E..*; VÁzouez, Fl.** \\ * Catedrático de Técnicas de Modificación de Conducta. Departamento de Psicología Clínica y Psicobiología. Universidad de Santiago de Compostela. \\ ** Profesor asociado en psicología clínica. Magister en Drogodependencias. Departamento de Psicología Clínica y Psicobiología. Universidad de Santiago de Compostela.
}

\section{Resumen:}

La relación entre la depresión y el consumo de tabaco se ha estudiado ampliamente en los adultos, mientras que en los adolescentes se le ha prestado poca atención. Sin embargo, del $40 \%$ al $70 \%$ de los adolescentes deprimidos desarrollan una comorbilidad adicional. En este artículo se revisa la prevalencia del consumo de cigarrillos, la prevalencia de la depresión y la relación entre la depresión y el consumo de cigarrillos en la dolescencia. Los estudios revisados sugieren un elevado grado de comorbilidad entre depresión y fumar cigarrillos en la adolescencia. Este hallazgo debería poner sobre aviso a los clínicos y a otros profesionales de la salud que hay una mayor probabilidad de depresión en los adolescentes fumadores y viceversa. La comorbilidad entre fumar y depresión en los adolescentes sugiere también que la prevención del hábito de fumar y los programas para dejar de fumar deben de tener en cuenta los porcentajes elevados de depresión en los jovenes adolescentes.

Palabras clave: fumar, depresión, adolescentes, tratamiento, prevención.

\begin{abstract}
:
The relationship between depression and tobacco consumption has been studied to a great extent in adults, where little attention has been given to same among adolescents. However, $40 \%$ to $70 \%$ of depressed adolescents developed additional comorbidity. We have reviewed the prevalence of cigarettes consumption, depression and, the relationship between depression and the consumption of cigarettes among adolescents. These finding shall be a serious warning to clinicians and other health professionals, that there is a higher probability of depression in adolescents who smoke and viceversa. The comorbidity between smoking and depression in adolescents suggests as well, that the prevention of the practice of smoking and smoking cessation programs should take into account the high levels of depression among adolescents.
\end{abstract}

Key words: smoking, depression, adolescents, treatment, prevention.

\section{INTRODUCCIÓN}

$\mathbf{E}$ n nuestra sociedad cada vez se observa una mayor preocupación respecto al tema del tabaco. La controversia y la sensibilización sobre los efectos que produce fumar cigarrillos en la salud son constantemente noticia desde ya hace algunos años en nuestro país. Si bien, en el mundo científico, ya hace más de tres décadas que se viene advirtiendo de lo perjudicial que es fumar (USDHHS, 1964), no es hasta hace unos pocos años cuando la mayoría de la opinón pública ha adoptado una actitud negativa sobre este hábito. De ser una conducta que se relacionaba con "cierto encanto, admiración, atractivo, sofisticación" ha pasado a ser considerado por la mayoría un hábito denostado, con connotaciones peyorativas.
Aun así, la conducta de fumar todavía se considera como la principal causa evitable de morbilidad y mortalidad (USDHHS, 1989). Fumar mata más personas cada año que el alcohol, otras drogas, homicidios, suicidio, accidentes ocasionados por vehículos de motor, incendios y SIDA, considerando todas estas causas de mortalidad en conjunto (Centers for Disease Control, 1991). Los beneficios que se obtienen al dejar de fumar son considerables. Dejar de fumar disminuye los riesgos de cáncer de pulmón, otros cánceres, ataques cardíacos, apoplejía y enfermedad pulmonar crónica (USDHSS, 1990). A pesar de ello, y aunque el consumo de tabaco ha disminuido, todavía continúa fumando el $36.2 \%$ de la población adulta española (Plan Nacional sobre Drogas, 1997) y la mayoría de los que intentan dejar de fumar no lo logran. Aunque 
aproximadamente el $60.0 \%$ de los fumadores españoles hacen un intento para dejar de fumar cada año (Ministerio de Sanidad y Consumo, 1992), menos del $11 \%$ de estos fumadores son capaces de mantenerse abstinentes al menos un año.

La identificación de intervenciones efectivas para dejar de fumar podrían fomentar que los fumadores intentasen abandonar los cigarrillos y aumentar los porcentajes de abstinencia, reduciendo de este modo la prevalencia de fumar y el sufrimiento, la enfermedad y las pérdidas económicas que produce el tabaco (Wetter et al., 1998). Sin embargo, aunque se ha dado un avance muy importante en la eficacia de los tratamientos del tabaquismo (American Psychiatric Association, 1996; Becoña y Vázquez, 1998a, 1998b), no ha sido todo lo que se hubiese deseado. Prevenir que los jóvenes lleguen a ser fumadores regulares podría ser una buena alternativa a los programas para dejar de fumar. Como concluye un informe reciente del Surgeon General (USDHHS, 1994), la adolescencia es la fase crucial de la vida para prevenir el consumo del tabaco y sus consecuencias porque es la etapa de la vida en la que se comienza a fumar, se llega a ser fumador regular y se adquiere la dependencia. Los estudios de prevalencia sobre el consumo de tabaco han puesto de manifiesto que al final de la adolescencia, la mayoría de los jóvenes que son fumadores lo seguirán siendo posteriormente.

Se han investigado una multitud de factores para determinar los antecedentes del consumo de cigarrillos entre los adolescentes (Becoña, Palomares y García, 1994; Botvin y Botvin, 1992; Miller y Slap, 1989). Algunos de ellos, tales como la conducta de fumar de los iguales y de los padres, cuentan con un apoyo sólido en la literatura científica (ver USDHHS, 1994). El conocimiento de algunos de estos factores, en concreto, la presión de los compañeros, fue una herramienta valiosa para ayudar a implementar estrategias de prevención adecuadas en los jóvenes para afrontar esta presión y, de este modo, conseguir que muchos de ellos no llegaran a fumar nunca. Sin embargo, hay un gran desconocimiento de los factores psicológicos, tales como la depresión, a pesar de que este tipo de factores parecen desempeñar un papel relevante en la progresión desde la fase de experimentación a la de fumador regular (Cleary, Hitchcock, Semmer, Flinchbaugh y Pinney, 1988). Entre los adultos fumadores, la prevalencia de trastornos psiquiátricos es mayor que en la población general, y la depresión es el trastorno psiquiátrico que más frecuentemente se ha asociado con fumar cigarrillos (Glassman, 1993). En general, se ha encontrado que los fumadores tienen mayores tasas de depresión mayor (ej., Breslau, Kilbey y Andreski, 1991) y síntomas de depresión (ej., Anda et al., 1990), aunque hay algunas excepciones a estos hallazgos (ej., Covey, Hughes, Glassman, Blazer y George, 1994)
La preocupación por estudiar la depresión en relación con el consumo de cigarrillos en la adolescencia es un hecho relativamente reciente (Vázquez y Becoña, 1998). Anteriormente los estudios de adolescentes han demostrado consistentemente diferencias en la salud mental de los fumadores adolescentes, pero en su mayor parte confiaron en medidas indirectas tales como la baja autoestima, ausencia de estrategias de afrontamiento adecuadas y neuroticismo. Un mejor conocimiento de los factores psicológicos específicos, que aumentan la vulnerabilidad de los jóvenes a fumar, puede estimular el interés en buscar estrategias mucho más eficaces de prevención. Por ello, es necesario profundizar y estudiar la relación de la conducta de fumar y la sintomatología depresiva en la adolescencia.

El objetivo de esta revisión es analizar en qué punto se encuentra la investigación sobre la relación entre fumar y depresión en los adolescentes y las implicaciones terapéuticas y preventivas que se derivan de ello.

\section{LOS ADOLESCENTES Y EL TABACO}

El número de adolescentes fumadores está aumentando en muchos países. Por ejemplo, en Estados Unidos, en 1995, aproximadamente el $63.5 \%$ de los jovenes, que estaban cursando el último año de instituto, informaron haber consumido tabaco en algún momento de sus vidas y el 34\% habían fumado durante los 30 días anteriores a la entrevista (Johnston, O’Malley y Backman, 1996).

La prevalencia diaria informada fue del 10\%, 18\% y $22 \%$ en los cursos octavo, décimo y décimo segundo, respectivamente (Johnston et al., 1996). Estos porcentajes todavía serían mayores si se hubiesen incluido a los estudiantes que abandonaron el Instituto. Pero todavía es más llamativo la evidencia que indica que el porcentaje en el octavo y décimo curso, que informaron que habían fumado durante los últimos 30 días, aumentó casi el 50\% desde 1991 a 1995 (Centers for Disease Control, 1996a). Pero lo más impactante son las estimaciones derivadas de estos hallazgos recientes, que indican que casi 5 millones de personas de 0 a 17 años en 1995 murieron prematuramente de una enfermedad relacionada con fumar (Centers for Disease Control, 1996b).

Desgraciadamente la situación del consumo de tabaco en adolescentes en España también es preocupante. En España, en un estudio de 1990, realizado por Mendoza, Sagrera y Batista (1994), dentro de un estudio más amplio que incluye diversos países, y que fue auspiciado por la Organización Mundial de la Salud, se encontró que a los 15 años el $11 \%$ de los chicos y el $15.5 \%$ de las chicas fumaban a diario, el 
$6.5 \%$ de los chicos y el $8.2 \%$ de las chicas fumaban una o más veces por semana y el $12.1 \%$ de los chicos y el $8.0 \%$ de las chicas fumaban menos de una vez por semana. Esto significa que un $29.6 \%$ de los chicos y un $31.7 \%$ de las chicas fumaban esporádica o diariamente. Dentro de los niveles escolares destacó el gran número de fumadores existentes en Formación Profesional I. El $41 \%$ de los chicos y el $46 \%$ de las chicas que estaban cursando el segundo curso de FP eran fumadores.

Casi todas las primeras pruebas con los cigarrillos ocurren con anterioridad a la finalización de los estudios de bachillerato (Centers for Disease Control, 1996a). Este hallazgo sugiere que si los adolescentes pudiesen mantenerse libres del tabaco, un porcentaje elevado de individuos nunca llegarían a fumar cigarrillos. La mayoría de los adultos que son fumadores probaron su primer cigarrillo a edades tempranas y llegaron a ser fumadores habituales a la edad de 18 años. El promedio de edad cuando experimentan con el primer cigarrillo suele rondar, de media, los 14.5 años. El promedio de edad cuando ellos llegaron a ser fumadores regulares es de 17.7 años. La adolescencia, por tanto, es el momento crítico en la vida del individuo para el comienzo y consolidación del consumo de tabaco.

Se estima que entre el $33 \%$ y el $50 \%$ de la gente joven que prueban sólo unos pocos cigarrillos llegan a ser fumadores regulares, un proceso que lleva un promedio de 2 a 3 años (Hirschman, Leventhal y Glynn, 1984). La adicción a la nicotina en los adolescentes es similar a la de los adultos, en cuanto que implica tolerancia a la nicotina, la necesidad de aumentar la cantidad fumada y la incapacidad para dejarlo. La mayoría de los adolescentes fumadores son adictos a la nicotina e informan que quieren dejar de fumar pero que son incapaces de lograrlo. De hecho, sólo un 20\% de los fumadores adolescentes regulares suelen dejar de fumar (USDHHS, 1994). Un porcentaje elevado de ellos experimentan el síndrome de abstinencia cuando intentan abandonar los cigarrillos (Rojas, Killen, Haydel y Robinson, 1998).

\section{LOS ADOLESCENTES Y LA DEPRESIÓN}

Aunque los investigadores llevan menos tiempo estudiando los trastornos del estado de ánimo en los niños y adolescentes que en adultos, sin embargo, en las últimas décadas hay una proliferación de actividad investigadora en el área de la depresión en la infancia y en la adolescencia. Se estima que la prevalencia puntual de la depresión mayor en los adolescentes oscila entre el 0.4\% y el 8.3\% (Birmaher et al., 1996).

La prevalencia de vida de depresión mayor se estima entre el $15 \%$ y el $29 \%$, un porcentaje similar a los datos que se obtienen con los adultos (Kessler et al.,
1994), que sugiere que la depresión en los adultos puede tener su origen en la adolescencia (Ciccheti y Toth, 1998, 1994). La prevalencia puntual de la distimia en los adolescentes oscila entre el $1.6 \%$ y el $8.0 \%$. Es más, en la adolescencia es más frecuente en una proporción de 2:1 en las chicas adolescentes que en los varones, proporción similar a la que se observa en los adultos (Culbertson, 1997; NolenHoeksema, 1990).

Las consecuencias de la depresión durante la adolescencia no pueden ser minimizadas (Cicchetti y Toth, 1998). Los trastornos depresivos no son acontecimientos que se puedan considerar como hechos normales en esta etapa de desarrollo ni problemas efímeros que desaparecen con el paso del tiempo (Kovacs, 1989). Incluso, cuando los episodios depresivos remiten, con frecuencia vuelven a aparecer e interfieren de modo muy importante en la vida de los jóvenes (Kovacs et al., 1984). Los chicos tienen un mayor riesgo para desarrollar posteriormente trastornos del estado de ánimo, cuando la distimia surge antes que la depresión. Por ejemplo, Kovacs, Akiskal, Gatsonis y Parrone (1994) encontraron que el 76\% de los chicos con un comienzo más temprano de distimia desarrollaron posteriormente un episodio de depresión mayor, y el 69\% desarrolló una depresión doble.

En la revisión de Birmaher et al. (1996) concluyeron que el promedio de un episodio de depresión mayor en los niños y en los adolescentes duraba de 7 a 9 meses. Aproximadamente el $90 \%$ de los episodios de depresión mayor remitían dentro de un período de dos años después de comenzar el episodio, mientras que los episodios restantes continuaban durante más tiempo. Con frecuencia la depresión mayor volvía a aparecer de nuevo en los chicos y en los adolescentes.

En comparación con la depresión mayor, la distimia tiene un curso mucho más prolongado, con un un promedio de 4 años de duración. Por lo general, los chicos con distimia sufren el primer episodio de depresión mayor 2 ó 3 años después del comienzo del episodio de distimia (Kovack et al., 1994).

Otro aspecto interesante de la depresión en los niños y adolescentes es la comorbilidad. Del $40 \%$ al $70 \%$ de los chicos y adolescentes deprimidos desarrollan un trastorno adicional. Se estima que del $20 \%$ al 50\% tienen 2 ó más trastornos adicionales. Los más frecuentes son los trastornos del estado de ánimo, los trastornos de ansiedad, los trastornos de conducta y el abuso de sustancias (Harrington, Rutter y Fombonne, 1996). En los niños y en los adolescentes la mayoría de los trastornos de ansiedad, normalmente el trastorno de ansiedad por separación, precede al episodio depresivo (Kovacs, 1996), mientras que en los adultos, el episodio depresivo precede al trastorno de ansiedad.

Normalmente la depresión mayor precede al comienzo del abuso del alcohol u otras sustancias, 
aproximadamente unos cuatro años antes (Birmaher et al., 1996). En general, los diagnósticos de comorbilidad aumentan el riesgo de depresión recurrente y afectan a la duración del episodio depresivo, intentos de suicidio, el buen estado general, respuesta al tratamiento y el uso de servicios de salud mental (Kovacs et al., 1994).

\section{DEPRESIÓNY CONSUMO DETABACO EN LA ADO- LESCENCIA}

La depresión es el diagnóstico más frecuente en los adultos que se encuentra asociado con el consumo de cigarrillos (Glassman, 1993). Como ya mencionábamos en la introducción, mientras la relación entre depresión y fumar se ha estudiado ampliamente en adultos (Covey, Glassman y Stetner, 1998; Glassman, 1993; Vázquez y Becoña, 1998), apenas se le ha prestado atención en los adolescentes. Los datos de algunos estudios indican que la depresión puede asociarse con el inicio de la conducta de fumar. Una importante cuestión en la compresión de esta asociación tiene que ver con la edad en la que se desarrolla la comorbilidad entre la depresión y la dependencia de la nicotina. La mayoría de los estudios (ej., Breslau et al., 1991) que han examinado esta cuestión se han basado en la población adulta de 20 o más años. Estos estudios no permiten determinar la edad en la que se establece una clara comorbilidad entre la depresión y la dependencia de la nicotina. Sin embargo, parece posible que ésta pueda ser establecida durante la adolescencia, porque sí está bien documentado el hecho de que las tasas de los trastornos depresivos y fumar cigarrillos se incrementan rápidamente de los 14 a los 18 años de edad (Angold, 1988; Miller y Slap, 1989; Patton et al., 1996).

Los datos de algunos estudios indican que la depresión puede asociarse con el consumo de cigarrillos (ver Tabla 1). En una investigación prospectiva (Kandel y Davis, 1986) se estudió una cohorte de 1.004 jóvenes adultos con un promedio de edad de 24.7 años, que participaron en un estudio que se había realizado nueve años antes. El estudio inicial, que se realizó de 1971 a 1972, se basó en una muestra aleatoria de adolescentes, representativa de la población perteneciente al sistema público de institutos del estado de Nueva York. De 1980 a 1981, nueve años después de ese primer contacto, se realizó una entrevista de seguimiento a una submuestra de aquellos adolescentes que habían participado en el estudio inicial cuando estaban cursando el equivalente a segundo y tercero de nuestro bachillerato. Una de las diferencias más importantes que se observaron en el estudio entre los jóvenes adultos que estaban deprimidos en la adolescencia y los que no lo estaban fue en el consumo de cigarrillos. La proporción de fumadores diarios en el último año fue del $57 \%$ entre los varones que habían sido clasificados como depresivos cuando estaban cursando sus estudios de bachillerato, mientras que en los que no estaban deprimidos fue del $30 \%$. La proporción de mujeres fumadoras fue del $50 \%$ en aquellas que tenían síntomas depresivos en su adolescencia, respecto al $34 \%$ de las que no habían experimentado dicha sintomatología. El estado de ánimo depresivo en la adolescencia predijo consistentemente ser fumador de cigarrillos entre los $24 \mathrm{y}$ los 25 años de edad, tanto para los hombres como para 24 y los 25 años de edad, tanto para los hombres como para las mujeres.

Stefanis y Kokkevi (1986) informaron de los hallazgos de un estudio a escala nacional sobre la población de estudiantes adolescentes que se centró en la asociación entre trastornos depresivos y uso de drogas. En este estudio, realizado en 1984, participaron 11.058 estudiantes adolescentes de una muestra de 96 escuelas representativas de los diferentes tipos de escuelas secundarias de Grecia. La edad de los jóvenes osciló entre 14 y 18 años de edad. Los resultados de este estudio proporcionaron una evidencia clara de una asociación entre el estado de ánimo deprimido y el uso de sustancias. Se observó un incremento de las puntuaciones en la escala de estado de ánimo deprimido cuando se pasaba de no usuario de ninguna sustancia legal o ilegal, al uso de sustancias legales (tabaco, alcohol, sustancias psicotrópicas prescritas y sin prescripción), a un uso de drogas ilegales (marihuana, psicodélicas, anfetaminas, cocaína) y a usuarios de heroína. Las chicas, pero no los chicos, que fumaban con más regularidad también tenían una puntuación mayor en la sintomatología depresiva que aquellas que no eran usuarias.

Otro estudio que se realizó con estudiantes de bachillerato fue el de Covey y Tam (1990). Llevaron a cabo un estudio sobre la relación entre síntomas depresivos y fumar dentro de un contexto de un modelo multifactorial. Para ello incluyeron 205 jóvenes (123 chicos y 82 chicas) alumnos que estaban realizando el curso equivalente a tercero de bachillerato en España, durante el año académico 1987-88. La media de edad fue de 16.1 años. Se encontraron puntuaciones más altas en la CES-D entre los estudiantes fumadores que entre los no fumadores.

Wang, Fitzhugh, Westerfield y Eddy (1994) estudiaron una muestra de 6.900 sujetos adolescentes (3.514 chicos y 3.386 chicas), de 14 a 18 años de edad, que seleccionaron del estudio "Informe de Actitudes y Prácticas de los Adolescentes" (Teenage Attitudes and Practices Survey). Éste había sido realizado por el Centro Nacional para la Estadística de la Salud (National Center for Health Statistics) en Estados Unidos en 1989. Entre la información de este informe se incluyeron medidas del estatus de fumador y de una 


\section{TABLA 1. La relación entre depresión y fumar en la adolescencia}

\begin{tabular}{|c|c|c|c|}
\hline Autores & Muestra & $\begin{array}{c}\text { Tipo de estudio } \\
\text { (transversal o prospectivo) }\end{array}$ & Trastornos estudiados \\
\hline Kandel y Davies (1996) & 1.004 & $\begin{array}{l}\text { Poblacional } \\
\text { (prospectivo) }\end{array}$ & Sintomatología depresiva \\
\hline Covey y Tam (1990) & 205 & $\begin{array}{l}\text { Poblacional } \\
\text { (transversal) }\end{array}$ & Sintomatología depresiva \\
\hline Wang et al. (1994) & 6.900 & $\begin{array}{l}\text { Poblacional } \\
\text { (transversal) }\end{array}$ & Sintomatología depresiva \\
\hline Patton et al. (1996) & 2.525 & $\begin{array}{l}\text { Poblacional } \\
\text { (transversal) }\end{array}$ & Sintomatología depresiva \\
\hline Wang et al. (1996) & 5.855 & $\begin{array}{l}\text { Poblacional } \\
\text { (prospectivo) }\end{array}$ & Sintomatología depresiva \\
\hline Escobedo et al. (1996) & 5.090 & $\begin{array}{l}\text { Poblacional } \\
\text { (transversal) }\end{array}$ & $\begin{array}{l}\text { Estado de ánimo deprimido } \\
\text { Historia de depresión mayor }\end{array}$ \\
\hline Fergusson et al. (1996) & 947 & $\begin{array}{l}\text { Poblacional } \\
\text { (prospectivo) }\end{array}$ & $\begin{array}{l}\text { Distimia } \\
\text { Depresión mayor }\end{array}$ \\
\hline Brown et al. (1996) & 1.709 & $\begin{array}{l}\text { Poblacional } \\
\text { (prospectivo) }\end{array}$ & $\begin{array}{l}\text { Depresión mayor } \\
\text { Historia de depresión mayor }\end{array}$ \\
\hline Choi et al. (1997) & 6.863 & $\begin{array}{l}\text { Poblacional } \\
\text { (longitudinal) }\end{array}$ & Sintomatología depresiva \\
\hline Escobedo et al. (1998) & 6.900 & $\begin{array}{l}\text { Poblacional } \\
\text { (prospectivo) }\end{array}$ & Sintomatología depresiva \\
\hline
\end{tabular}

serie de factores relacionados con síntomas depresivos. Wang et al. (1994) analizaron esta información y encontraron que los fumadores adolescentes era más probable que los no fumadores que experimentaran síntomas asociados de depresión tales como sentimientos de infelicidad, tristeza o depresión, desesperanza sobre el futuro y problemas de sueño.

Ya más recientemente, se sigue encontrado apoyo a dicha relación en la adolescencia. Patton et al. (1996) realizaron un estudio cuyo objetivo fue examinar la asociación de fumar con la depresión y la ansiedad en una muestra representativa de 2.525 adolescentes fumadores de Victoria (Australia). El $48.2 \%$ de los adolescentes eran hombres y el $51.8 \%$ mujeres, con una edad promedio de 15 años y un rango de edad de 11 a 17 años. Todas las subescalas del inventario de entrevista clínica (DIS: Clinical Interview Schedule) mostraron asociaciones significativas entre fumar regularmente y las dimensiones de psicopatología que recoge el inventario. La depresión fue la segunda asociación más significativa que se encontró en el análisis de los datos.

Los participantes en el estudio del Informe de Actitudes y Prácticas de los adolescentes norteamerica- nos fueron entrevistados de nuevo en 1993. Wang, Fitzhugh, Turner, Fu y Westerfield (1996) seleccionaron una muestra de 5.855 sujetos que participaron en 1989 (Tiempo 1) y en 1993 (Tiempo 2). Estos autores no pudieron determinar la dirección de la causalidad de la relación entre fumar y depresión. Sin embargo, los datos sugieren que la relación entre ambos problemas es recíproca.

Escobedo, Kirch y Anda (1996) evaluaron la relación entre la depresión mayor y el estado de ánimo deprimido y el inicio de la conducta de fumar entre una población de 5.090 jóvenes de ascendencia mejicana, puertorriqueña y cubana $(66.8 \%$ varones y el $38.6 \%$ mujeres). Utilizaron los datos del Informe de Investigación de Nutrición y Salud Hispana (Hispanic Health and Nutrition Examination Survey) que se recogieron entre 1982 y 1984 en cinco estados del suroeste de Estados Unidos (California, Arizona, Nuevo México, Colorado y Texas). Encontraron que tener un estado de ánimo depresivo, una historia de depresión mayor o ambas condiciones estaba asociado con el riesgo de iniciarse en la conducta de fumar durante la infancia, la adolescencia y en los adultos jóvenes (la probabilidad de haber sido alguna vez fumador fue mayor para 
aquellos que tenían un estado de ánimo depresivo, una historia de depresión mayor o ambas cosas). A su vez, los sujetos con una historia de depresión mayor tenían más probabilidad de haber fumado alguna vez que los que experimentaron estados de ánimo deprimido.

Fergusson, Lynskey y Horwood (1996) estudiaron la comorbilidad entre trastornos depresivos y dependencia de la nicotina en una cohorte de jóvenes de 16 años de Nueva Zelanda. Los objetivos del estudio fueron documentar el alcance de la comorbilidad entre la depresión y la dependencia de la nicotina a las edades de 15 y 16 años y el grado en el que una serie de factores de riesgo podría explicar esa comorbilidad. Los datos los extrajeron de una cohorte de 947 jóvenes de Nueva Zelanda que fueron seguidos desde su nacimiento hasta los 16 años. Se encontraron asociaciones significativas entre la dependencia de la nicotina y los trastornos afectivos. Se detectó una comorbilidad de moderada a elevada entre la depresión y la dependencia de la nicotina a los 16 años. Los jóvenes que padecían trastornos depresivos presentaban una odds ratio de la dependencia de la nicotina que era más de 4.5 veces mayor que la odds ratio de los que no tenían trastornos depresivos.

Brown, Lewinsohn, Seeley y Wagner (1996) estudiaron la relación de fumar cigarrillos y el trastorno de depresión mayor y otros trastornos psiquiátricos, tranversal y prospectivamente. Utilizaron en el estudio una muestra representativa de 1.709 (48\% hombres y 52\% mujeres) adolescentes que estaban estudiando el equivalente a los cursos de bachillerato en España. La media de edad de la muestra fue de 16.6 años (14 a 18 años). Los resultados sugirieron importantes relaciones entre fumar cigarrillos y los distintos trastornos psiquiátricos estudiados, especialmente con la depresión mayor. En los hallazgos prospectivos encontraron, que los que eran fumadores cuando se realizó la primera entrevista, tenían un riesgo dos veces mayor de desarrollar un episodio de depresión mayor en el seguimiento de los 12 meses (segunda entrevista) que los no fumadores. De todos los trastornos que analizaron, sólo la prevalencia de vida de depresión mayor fue factor de riesgo para el comienzo de fumar (IC 95\%; Odds ratio $=2.04, p<0.05)$. Sin embargo, en los análisis transversales no encontraron una comorbilidad significativa entre fumar y depresión mayor.

Otro estudio en el que se analizó si fumar cigarrillos en la adolescencia predice el desarrollo de síntomas depresivos fue el de Choi et al. (1997). Utilizaron una muestra de 6.863 adolescentes (12-18 años) norteamericanos. Llevaron a cabo dos evaluaciones. En la segunda evaluación (un año después de la primera evaluación) encontraron que el 11.5\% había desarrollado una sintomatología depresiva importante. Había diferencias significativas entre las chicas (15.3\%) y los chicos $(8.1 \%)$.
Escobedo, Reddy y Giovino (1998) también analizaron los datos del estudio prospectivo del Informe de Actitudes y Prácticas de los Adolescentes. Para estudiar la relación entre síntomas depresivos y fumar, Escobedo et al. (1988) incluyeron 7.885 jóvenes adolescentes entre 12 y 18 años de edad, que habían participado en ese estudio. Encontraron que los adolescentes con síntomas depresivos era más probable que empezaran a fumar que los adolescentes no deprimidos (Odds ratio $=1.3$ ). Los porcentajes de síntomas depresivos entre no fumadores fue del 13\% y en los fumadores del $20 \%$.

\section{IMPLICACIONES CLÍNICASY PREVENTIVAS}

Como hemos visto hasta aquí, existe una clara relación entre el consumo de cigarrillos en los adolescentes y la depresión. Aunque todavía hay pocos estudios, los que hay sugieren unívocamente que está bien establecida una comorbilidad entre el consumo de cigarrillos y la depresión en la adolescencia. Estudios prospectivos (ej., Escobedo et al., 1998; Kandel y Davis, 1986) y transversales (ej., Covey y Tam, 1990; Wang et al., 1994) han demostrado que hay una sólida relación entre fumar en la adolescencia y sintomatología depresiva. Otros estudios han demostrado que también hay relación entre depresión mayor (ej., Brown et al., 1996) e historia de depresión mayor (ej., Escobedo et al., 1996) y fumar. La relación también se encontró cuando se evaluó la asociación entre depresión y dependencia de la nicotina (ej., Fergusson et al., 1996).

La evidencia de una relación bidireccional entre depresión e iniciación de la conducta de fumar lla depresión es un factor de riesgo para comenzar a fumar y fumar es un factor de riesgo para sufrir depresión), sugiere la posibilidad de que pueda haber una vulnerabilidad que es común a ambos trastornos. Fergusson et al. (1996) encontraron factores de riesgo comunes, tales como la afiliación con iguales delincuentes y la baja autoestima, que también explicaron la comorbilidad entre la depresión y la dependencia a la nicotina. Estos hallazgos son consistentes con las conclusiones de Breslau, Kilbey y Andreski (1993), Breslau, Peterson, Schultz, Chilcoat y Andreski (1998) y Kendler et al. (1993) de que la asociación entre la dependencia de la nicotina y la depresión es, en su mayor parte o completamente, no causal y probablemente reflejen factores antecedentes que están asociados con ambas condiciones. Sin embargo, Kendler et al. (1993) concluyeron que los factores que conducían a esta comorbilidad eran probablemente genéticos, mientras que en el estudio de Fergusson y colaboradores se sugiere que son factores sociales $y$ de la infancia los que explican una parte importante de 
esa asociación. Un dato que necesita ser investigado es que en el estudio de Brown et al. (1996) encontraron esa bidireccionalidad entre depresión y fumar, pero paradójicamente en los análisis transversales no encontraron una relación significativa entre fumar y depresión. Estos autores sugieren como una posible explicación que la relación entre depresión y fumar exista sólo en los fumadores que tienen realmente un problema de dependencia de la nicotina.

Por tanto, los estudios de adolescentes sugieren que la depresión juega un papel importante en la iniciación del consumo (ej., Brown et al., 1996; Escobedo et al., 1998; Kandel y Davis, 1986). Como resultado de estos hallazgos, se ha sugerido que las personas con trastornos depresivos pueden ser especialmente vulnerables a la adicción de la nicotina. Sin embargo, ésta es una cuestión sobre la que todavía hay que profundizar. La mayor dificultad aparece en el diagnóstico de depresión en la adolescencia, puesto que la mayor parte de los adolescentes parecen experimentar algún tipo de sintomatología depresiva durante este período (Calafat, Amengual, Mejías, Borrás y Escosura, 1990), que aparece junto a síntomas de ansiedad o inquietud. A estos efectos, parecería interesante, a esta edad especialmente, distinguir entre depresión clínicamente instaurada y sintomatología depresiva ligada a esa etapa de desarrollo, estableciendo con mayor precisión el peso que tiene la depresión y la depresividad, en terminos de Calafat y Amengual (1991), en el inicio y consolidación del consumo de cigarrillos.

Otra faceta interesante es descubrir las conexiones que tiene la depresión con otros factores personales, circunstancias familiares, grupos de amigos, eventos vitales y la interrelacion entre todos ellos. El que fumen los padres es un factor de riesgo bien demostrado para fumar en la adolescencia (ver USDHHS, 1994) y es predictivo de mayores tasas de depresión en sus hijos (Burke, Burke, Regier y Raes, 1990). El hábito de fumar en los padres presenta una asociación independiente con fumar en la adolescencia, con una influencia clave en la transición a fumar regularmente. Los teóricos del aprendizaje social han argumentado que el desarrollo de fumar está fuertemente influenciado por los compañeros y la necesidad de establecer vínculos estrechos con los compañeros. Una persona joven con un nivel elevado de síntomas depresivos y asociado a una baja autoestima y autoconfianza podría ser particularmente vulnerable a las influencias de fumar del grupo de los iguales. De modo similar, los mensajes de los anuncios que vinculan fumar con el atractivo sexual, buena forma física e independencia tienen atractivo para una persona con una pobre autoimagen.

Por otra parte, el elevado grado de comorbilidad entre depresión y fumar cigarrillos debería poner sobre aviso a los clínicos y a otros profesionales de la salud que hay una mayor probabilidad de depresión en los adolescentes fumadores y viceversa. Los estudios analizados en esta revisión sugieren que cuando un adolescente fuma cigarrillos tiene un riesgo elevado de depresión. Por tanto, es recomendable incluir la evaluación del consumo de cigarrillos en la evaluación clínica. Hace tiempo que conocemos las consecuencias adversas de fumar para la salud física (USDHHS, 1964). El resultado de esta revisión nos indica que también hay importantes consecuencias para la salud mental.

La comorbilidad entre fumar y depresión en los adolescentes sugiere también que la prevención de fumar y los programas para dejar de fumar necesitan tener en cuenta los porcentajes elevados de un trastorno adicional de depresión en los jóvenes fumadores. Quizás esto pueda explicar que los primeros programas de prevención centrados en el conocimiento, actitudes y creencias sobre fumar y los efectos dañinos sobre la salud no obtuvieran buenos resultados (Becoña et al., 1994). Los programas de prevención más recientes que se centran fundamentalmente en enseñar estrategias para resistir las influencias sociales a fumar $y$ sobre habilidades para aumentar la competencia individual en general están obteniendo resultados más prometedores (Botvin y Botvin, 1992). A la luz de los hallazgos de esta revisión, los programas futuros de prevención deberían considerar la posibilidad de incorporar estrategias para afrontar el afecto negativo e intensificar el afecto positivo. Intervenciones clínicas que hayan demostrado ser eficaces en el tratamiento de la depresión en adolescentes (ej., Lewinsohn, Clarke, Hops y Andrews, 1990) se podrían adaptar para este propósito, de forma similar a lo que se ha hecho para los adultos (Hall, Muñoz y Reus, 1994).

Quizás ya es necesario dar otro paso y se necesiten diseñar e implementar programas especializados dirigidos a subgrupos específicos de fumadores adolescentes, como por ejemplo, el de los adolescentes con depresión, historia de depresión mayor o sintomatología depresiva.

\section{REFERENCIAS BIBLIOGRÁFICAS}

American Psychiatric Association (1996). Practice guideline for the treatment of patients with guideline for the treatment of patients with nicotine dependence. American Journal of Psychiatry, 153, 1-31.

Anda, R.F., Williamson, D.F., Escobedo, L.G., Mast, E.E., Giovino, G.A. y Remington, P.L. (1990). Depression and the dynamics of smoking: A national perspective. JAMA, 264, 15411545.

Angold, A. (1988). Childhood and adolescent depression. I. Epidemiological and etiological aspects. British Journal of Psychiatry, 152, 601-617.

Becoña, E., Palomares, A. y García, M.P. (1994). Prevención y tratamiento del tabaquismo. Madrid: Pirámide. 
Becoña, E. y Vázquez, F.L. (1998a). Estado actual de las alternativas terapéuticas para dejar de fumar. Adicciones, 10, 69-83.

Becoña, E. y Vázquez, F.L. (1998b). Tratamiento del tabaquismo. Madrid. Dykinson.

Birmaher, B., Ryan, N., Williamson, D., Brent, D., Kaufman, J., Dahl, R., Perel, J. y Nelson, B. (1996). Childhood and adolescence depression: A review of the past 10 years. Part I. Journal of the American Academy of Child and Adolescent Psychiatry, 35, 1427-1439.

Botvin, G.J. y Botvin, E.M. (1992). Adolescent tobacco, alcohol, and drug abuse: Prevention strategies, empirical findings, and assessment issues. Developmental and Behavioral Pediatrics, 13, 290-301.

Breslau, N., Kilbey, M. y Andreski, P. (1991). Nicotine dependence, major depression and anxiety in young adults. Archives of General Psychiatry, 48, 1069-1074.

Breslau, N., Kilbey, M. y Andreski, P. (1993). Nicotine dependence and major depression: New evidence from a prospective investigation. Archives of General Psychiatry, 50, 31-35.

Breslau, N., Peterson, E.L., Schultz, L.R., Chilcoat, H.D. y Andreski, P. (1998). Major depression and stages of smoking. A longitudinal investigation. Archives of General Psychiatry, 55, 161-166.

Brown, R.A., Lewinsohn, P, Seeley, J.R. y Wagner, E.F. (1996). Cigarette smoking, major depression, and other psychiatric disorders among adolescents. Journal of the American Academy of Child and Adolescent Psychiatry, 35, 1602-1610.

Burke, K.C., Burke, J.D., Regier, D.A. y Raes, D.S. (1990). Age at onset of selected mental disorders in five community populations. Archives of General Psychiatry, 47,511518

Calafat, A. y Amengual, M. (1991). Depresión, depresividad y toxicomanía. Adicciones, 2, 75100.

Calafat, A., Amengual, M., Mejías, G., Borrás, M. y Escosura, M.J. (1990). Los jóvenes de hoy. Palma de Mallorca: Consell Insular de Mallorca.

Centers for Disease Control. (1991). Smoking-attributable mortality and years of potential life lost: United States, 1990. Morbidity and Mortality Weekly Report, 40, 6263, 6971.

Centers for Disease Control. (1996a). Tobaco use and usual source of cigarettes among high school students- United States. Morbidity and Mortality Weekly Report, 45, 413-418.

Centers for Disease Control. (1996b). Projected smokingrelated death among youth: United States. Morbidity and Mortality Weekly Report, 45, 971-974.

Choi, W.S., Patten, C.A., Gillin, J.C., Kaplan, R.M. y Pierce, J.P. (1997). Cigarette smoking predicts development of depressive symptoms among U.S. Annals of Behavioral Medicine, 19, 42-50.

Cicchetti, D. y Toth, S.L. (1998). The development of depression in children and adolescents. American Psychologist, $53,221-241$.
Cleary, P.D., Hitchocok, J.L., Semmer, N., Flinchbaugh, L.J. y Pinney, J.M. (1988). Adolescence smoking: Research and health politics. Milbank Quarterly, 66, 137-171.

Covey, L.S., Hughes, D., Glassman, A.H., Blazer, D.G. y George, L.K. (1994). Eversmoking, quitting, and psychiatric disorders: Evidence from the Durham, North Carolina, Epidemiologic Catchment Area. Tobacco Control, 3, 222-227.

Covey, L.S., Glassman, A.H. y Stetner, F. (1998). Cigarette smoking and major depression. Journal of Addictive Diseases, $17,35-46$.

Covey, L.S. y Tam, D. (1990). Depressive mood, the singleparent home, and adolescent cigarette smoking. American Journal of Public Health, 80, 1330-1333.

Culbertson, F.M. (1997). Depression and gender. An international review. American Psychologist, 52, 25-31.

Escobedo, L., Kirch, D.G. y Anda, R.F. (1996). Depression and smoking initiation among US Latinos. Addiction, 91, 113-119.

Escobedo, L., Reddy, M. y Giovino, G. (1998). The relationship between depressive symptoms and cigarette smoking in US adolescents. Addiction, 93, 433-440.

Fergusson, D., Lynskey, M. y Horwood, J. (1996). Comorbidity between depressive disorders and nicotine dependence in a cohort of 16-year-olds. Archives of General Psychiatry, 53, 1043-1047.

Glassman, A.H. (1993). Cigarette smoking: Implications for psychiatric illness. American Journal of Psychiatry, $150,546-553$

Johnston, L.D., O’Malley, P.M. y Backman, J.G. (1996). National Survey Results on Drug Use From the Monitoring the Future Study: 19751995. Rockville, MD: National Institute on Drug Abuse.

Hall, S.M., Muñoz, R.F. y Reus, V.I. (1994). Cognitive-behavioral intervention increases abstinence rates for depressive-history smokers. Journal of Consulting and Clinical Pschology, 62, 141-146.

Harrington, R., Rutter, M. y Fombonne, E. (1996). Developmental pathways in depression: Multiple meanings, antededents, and endpoints. Development and Psychopathology, 8, 601-616.

Hirschman, R.S., Leventhal, H. y Glynn, K. (1984). The development of smoking behavior: Conceptualization and supportive cross-sectional survey data. Journal Applied Social Psychology, 14, 184-206.

Kandel, D.B. y Davies, M. (1986). Adult sequelae of adolescent depressive symptoms. Archives of General Psychiatry, 43, 255-262.

Kendler, K.S., Neale, M.C., MacLean, C.J., Heath, A.C., Eaves, L.J. y Kessler, R.C. (1993). Smoking and major depression: A causal analysis. Archives of General Psychiatry, 50, 3643.

Kessler, R., McGonagle, K., Zhao, S., Nelson, C., Hughes, M., Eshleman, S., Wittchen, H. y Kendler, K. (1994). Lifetime and 12 month prevalence of DSMIII-R psychiatric disorders in the United States: Results from the national comorbidity survey. Archives of General Psychiatry, 51, 819. 
Kovacs, M. (1989). Affective disorders in children and adolescents. American Psychologist, 44, 209-215.

Kovacs, M. (1996). Presentation and course of major depressive disorder during childhood and later years of the life span. Journal of the American Academy of Child and Adolescent Psychiatry, 35, 705-715.

Kovacks, M., Akiskal, H., Gatsonis, C. y Parrone, P. (1994). Childhood-onset dysthymic disorder: Clinical features and prospective naturalistic outcome. Archives of General Psychiatry, 51, 365-374.

Kovacs, M. , Feinberg, T., Crouse-Novak, M., Paulauskas, S., Pollock, M. y Finkelstein, R. (1984). Depressive disorders in childhood. II. A longitudinal study of the risk for a subsequent major depression. Archives of General Psychiatry, 41, 643649.

Lewinsohn, P.M., Clarke, G.N., Hops, H., Andrews, J.A. (1990). Cognitive-behavioral treatment for depressed adolescents. Behavior Therapy, 21, 385-401.

Mendoza, R., Sagrera, R.R. y Batista, J.M. (1994). Conductas de los escolares españoles relacionadas con la salud (1986-1990). Madrid: CSIC.

Miller, S.K. y Slap, G. (1989). Adolescent smoking: A review of prevalence and prevention. Journal of Adolescent Health Care, 10, 129-135.

Ministerio de Sanidad y Consumo (1992). Estudio de los estilos de vida de la población adulta española. Madrid: Ministerio de Sanidad y Consumo. Dirección General de Salud Pública.

Nolen-Hoeksema, S. (1990). Sex differences in depression. Standford, CA: Standford University Press.

Patton, G.C., Hibbert, M., Rosier, M.J., Carlin, J.B., Caust, J. y Bowes, G. (1996). Is smoking associated with depresssion and anxiety in teenagers? American Journal of Public Health, 86, 225-230.

Plan Nacional sobre Drogas (1997). Encuesta domiciliaria sobre uso de drogas. Madrid: Plan Nacional sobre Drogas.
Rojas, N.L., Killen, J.D., Haydel, K.F. y Robinson, T.N. (1998). Nicotine dependence among adolescent smokers. Archives of Pediatrics and Adolescent Medicine, 152, 151156

Stefanis, C.N. y Kokkevi, A. (1986). Depression and drug use. Psychopathology, 19, 124-131.

USDHHS (1964). Smoking and Health. Report of Advisory Committee to the Surgeon General of the Public Health Services. Rockville, Maryland: U.S. Department of Public Health Service.

USDHHS (1989). The health consequences of smoking. $\mathbf{2 5}$ years of progress. A report of the Surgeon General. Rockville, MD: U.S. Department of Health and Human Services.

USDHHS (1990). The health benefits of smoking cessation. A report of the Surgeon General. Washington, DC: U.S. Department of Health and Human Services.

USDHHS (1994). Preventing tobacco use among young people. A report of the Surgeon General. Atlanta, Georgia: U.S. Department of Health and Human Services.

Vázquez, F. y Becoña, E. (1998). ¿El hábito de fumar tiene relación con la depresión? Psicothema, 10, 229-239.

Wang, M.Q., Fitzhugh, E.C., Turner, L., Fu, Q. y Westerfield, R.C. (1996). Association of depressive symptoms and school adolescents' smoking: A crosslagged analysis. Psychological Reports, 79, 127130.

Wang, M.Q., Fitzhugh, E.C., Westerfield, R.C. y Eddy, J.M. (1994). Predicting smoking status by symptoms of depresssion for U.S. Adolescents. Psychological Reports, 75, 911914.

Wetter, D.W., Fiore, M.C., Gritz, E.R., Lando, H.A., Stitzer, M.L., Hasselblad, V. y Baker, T. (1998). The Agency for Health Care Policy and Research Smoking Cessation Clinical Practice Guideline. American Psychologist, 53, 657669. 
